\title{
Numerical Inductor Optimization
}

\author{
Axel von der Weth and J. Aktaa \\ Institut für Materialforschung II, Forschungszentrum Karlsruhe, P.O. Box 3640, D-76021 Karlsruhe
}

This paper shows a method for the numerical quality factor optimization of a high frequency inductor based on a genetic algorithm. This is pointed out by an example of a trench-type or I-inductor. A comparison between different design approaches will show the advantages of themselves. First results for a further application as a micro transformer of such a device are described.

Key words: Inductor, quality factor optimization, genetic algorithm, inductor simulation, micro transformer

\section{Introduction}

For the application of any micro system it is important to ask for the limits of its performance depending on the parameters of the fabrication technology. The experimental answer of this question might be expansive or in some cases unattainable. This causes to choose a numerical method.

The first topic of the current paper will answer such a question for the case of a trench-type ${ }^{1)}$ inductor called below I-inductor ${ }^{2}$. The performance of any inductor is given by the quality factor. Losses from ohmic resistance and capacitance have to be considered for a first order approach in the case of an I-inductor. Main goal of optimization of any inductor is to achieve the maximum possible quality factor together with the fitting dimensions of the inductor. This task will be solved using genetic algorithms. The results of our calculations foretell interesting quality factors for industrial realization of the
I-inductor.

The second topic will contain first results of simulations of a micro transformer. A concept for such a device is shown using design rules and results from the first topic.

\section{Inductor type}

The different inductor types considered in this paper are thin film micro inductors, which consists of two conducting metallic layers with a magnetic film in between. These three films are isolated from each other by dielectric films $\left(\mathrm{SiO}_{2}\right)$. Structuring of the metallic layers yields the strip conductors, which are connected from one layer to the other layer by wire wholes or vias. A promising candidate for a CMOS-integrated inductor might be the I-Inductor ${ }^{3), 4)}$. An I-inductor, Fig. 1 and Fig. 2, consists of at least two anti parallel driven strips or cores reasonably ${ }^{2)}$ made of a magnetic material with an uniaxial anisotropy ${ }^{5)}$. The hard axis is oriented in $\mathrm{x}$-direction. Therefore the relative permeability in $\mathrm{x}$ direction $\mu_{\mathrm{x}}$ is large in the high frequency case, while $\mu_{\mathrm{y}}$ and $\mu_{\mathrm{z}}$ are equal to unity. These strips are magnetically anti parallel excited by solenoidal like winding system. The main advantages of this type of inductor are the suitability for a magnetic material with uniaxial anisotropy and a negligible eddy current excitation in the substrate. Other advantages and restriction of this type has been discussed in reference 6).

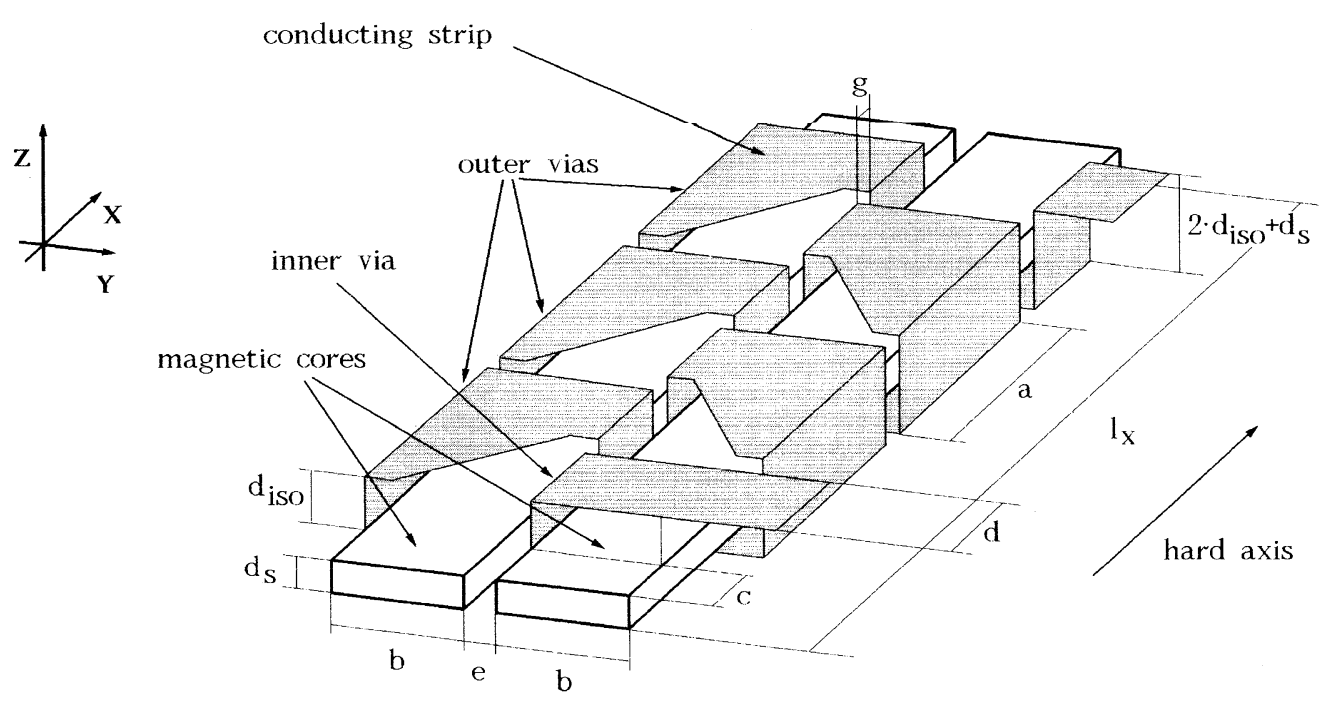

Fig. 1 Sketch of an I-inductor using two cores, trapezoid and rectangle shaped conducting strips. 


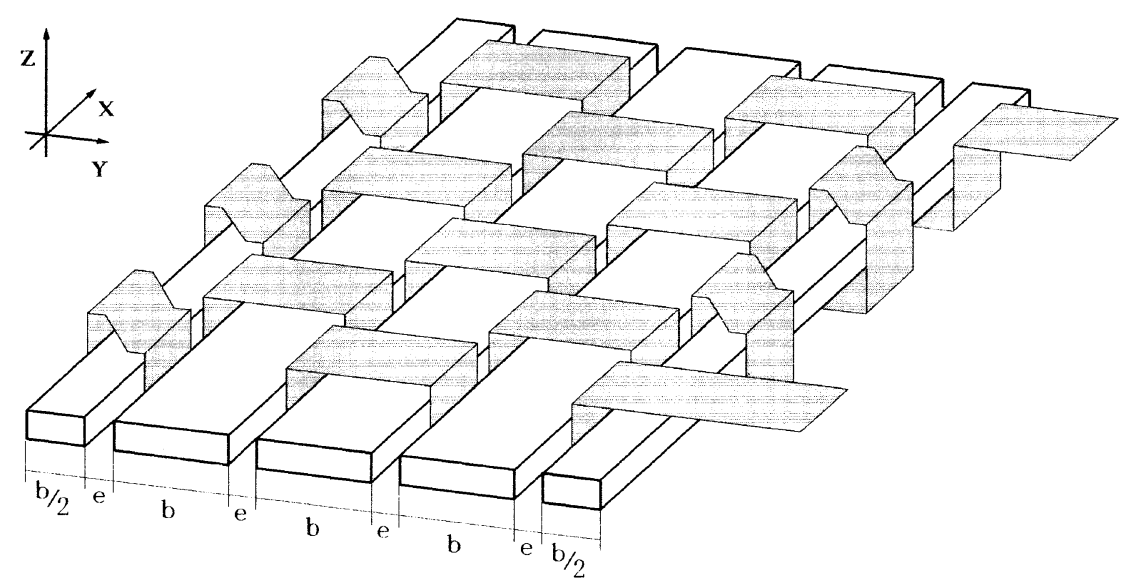

Fig. 2 I-inductor using five magnetic cores, rectangle and parallelogram shaped conducting strips.

\section{Inductor optimization}

Our numerical inductor optimization has to lead to the maximum possible quality factor depending on the design of the device considering structure and material parameters. This will performed by varying geometrical dimension of the inductor e.g. length, width, number of cores and shape of the conducting elements for solenoid system, see Fig. 1 and 2. The input data needed are the structural parameters like minimum possible width between adjacent parts of the inductor, maximum possible film thickness of all layer and the core material parameters conductivity and permeability.

\subsection{Inductor model}

Basic steps of any inductor optimization are numerical models ${ }^{2), 3), 6)}$ for the quality factor $Q$ of the devices. A basic approach for the quality factor like eq. (1) fits only if the frequency is far less than self resonance of the device.

$$
Q=2 \pi f L / R
$$

The optimized inductor application do not fulfill this condition. It is impossible to simulate the well known frequency dependent quality factor maximum by eq. (1).

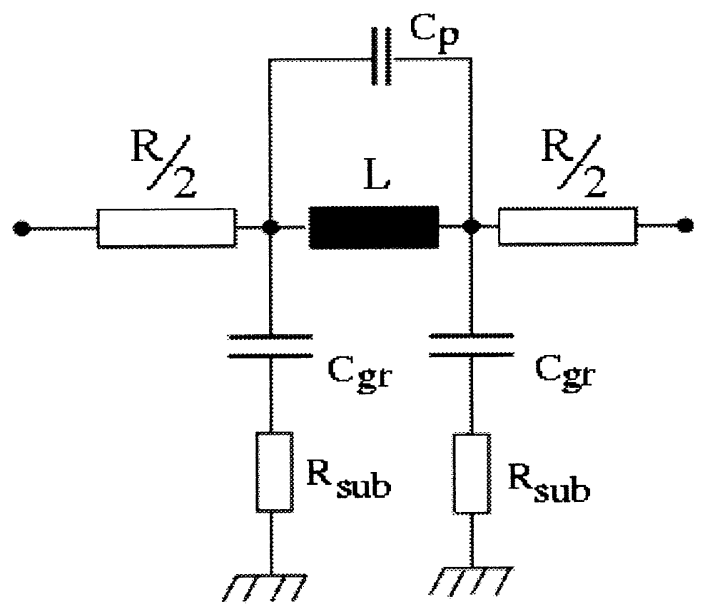

Fig. 3 Equivalent circuit diagram.
This causes us to use an equivalent circuit diagram as shown in Fig. 3. This diagram represents losses by resistors and capacitors. Their resistances can be calculated by thickness of the (aluminum) metallic layers $(1 \mu \mathrm{m}$ obtained for both metallic layer) and geometrical dimensions of the conducting elements. A non ideal galvanic couple between the different aluminum layers by the vias is considered by a doubled ohmic resistance of the vias in contrast to our earlier calculations ${ }^{6)}$. The capacitances $c_{\mathrm{p}}$ and $c_{g r}$ are estimated by both the effective areas and their distance between. The complete area of the inductor is used for the calculation of the capacitance (equal to $2 c_{\mathrm{gr}}$ in Fig. 3) between the conducting substrate and the inductor by assuming a dielectric layer of $1 \mu \mathrm{m}$ thickness. The magnetic core as a conductor inside windings occupies the area of all windings and $d_{\text {iso }}$, see Fig. 1, for the estimation of the capacitance parallel to the inductance ( $c_{\mathrm{P}}$ in Fig. 3 ). There is no need for the consideration of stray capacitances of $c_{\mathrm{gr}}$ and $c_{\mathrm{p}}$, because the longitudinal dimensions of all areas are much larger than the thickness of the dielectric films. The inductance is calculated by use of the FEM based solver for the Maxwell's equations "MAFIA" reducing the problem to a static one. This is justified by the small dimensions of the device (far less than the wavelength), the absence of skin effect in the thin strip conductors (thickness $1 \mu \mathrm{m}$ ) and the absence of eddy current excitation inside the magnetic cores $\left(d_{\mathrm{s}}=1.3 \mu \mathrm{m}\right.$ uncritical film thickness from measurement $\left.{ }^{5}\right)$ and the substrate ${ }^{6}$. The minimum structural length for the conducting strips of more than $1 \mu \mathrm{m}$ allows to neglect proximity effects, and lowers the requirements for the equipment of realization. Now for the calculation of the inductance meshes are used with up to $1.3 \times 10^{7}$ elements, because the lateral dimensions are very large (total size of the device $28 \mu \mathrm{m}$ to $1000 \mu \mathrm{m}$ ) in contrast to the thickness of about $1 \mu \mathrm{m}$ of each film premised ideal planar. It is important to consider the magnetic material anisotropy. Such a procedure would not be fast enough for the 
following steps. Therefore a semi-analytical solutions together with an anisotropic linear material model is used for the inductance estimation ${ }^{2)}$, which bases on the results from "MAFIA". After calculation of all magnitudes of the complex impedance in the equivalent circuit diagram has to be estimated dependent on the frequency $(f=0.9 \mathrm{GHz})$. The quality factor is given as quotient of imaginary and real part of the complex impedance.

\subsection{Genetic algorithm}

It is well known from macroscopic inductors that the maximum achievable quality factor goes to first order reverse with the inductance: For instance if the number of turns is doubled assuming two optimized magnetically coupled inductors (like Fig. 5, primary and secondary coil connected in series), the ohmic resistance $R$ is increased by a factor of two and the inductance $L$ by a factor of four. The capacitances are increased by a factor of two. The last two properties lower the maximum operating frequency at least by a factor of 0.35 (self resonance frequency dropped down). Inserting this into eq. (1) yields a reduction for the quality factor of at least $30 \%$. This causes the "inalienable" importance for to be compared inductors to posses the same inductance with different e.g. geometrical dimensions, later called target inductance. The numerical and semi-analytical approximations described in previous section enable only a fast calculation of the quality factor and the inductance as a function of the dimensions. But the reverse map is needed. This problem is solved by a genetic algoritm ${ }^{6}$ : The algorithms chooses with a random generator reasonable input data set, which consists of all geometrical dimensions and number of turns.

\subsection{Inputs to the genetic algorithm}

Some of the dimensions are held constant or are dependent from other dimensions: As less as the distance between adjacent cores is decreased, the inductance is increased, size of the device and capacitance are decreased. But requirements to a later processing of the device are growing. For instance some area for the vias (possibly funnel shaped after realization and wider in the upper metallic layer) and a distance about $1 \mu \mathrm{m}$ between via and magnetic cores is needed. This causes a distance between adjacent core of $e=4 \mu \mathrm{m}$. There had been found in earlier simulations to set the length $c$ (Fig. 1) of core part, which sticks out of the solenoid winding system, as large as the width $b$ of the core. Longer cores $(c>b$, Fig. 1) do not raise up the inductance reasonably, but such cores increase the capacitance between ground and core. Shorter cores lower the inductance. Of course the distance between adjacent inner vias inside the magnetic core or strip system is set to the minimum structure length $g$. With these restriction the core length $l_{\mathrm{x}}$ is given by the number of solenoid turns $n$ and the length of a via $d$.

\subsection{Flow of operation}

The algorithm proofs first physical restrictions like a violated minimum possible width, maximum possible core length ${ }^{6}$. If one condition is not fulfilled the algorithm chooses a new set of input data otherwise the inductance is calculated and compared with the target inductance. This is called try. If the estimated inductance is in the range of the target inductance (error 10\%), the quality factor is calculated and one solution is found. The algorithm will find after some tries a new inductor with a fitting inductance. In the case of a higher quality factor of the new solution the new inductor is stored as "best" inductor. Note, there are two optimization strategies for this problem: First quality optimization at fixed frequency $f$, which is used here. Second it is possible to select a set of input parameter, for which the frequency derivative of the quality factor at this fixed frequency is vanishing. Maximum possible quality factors are observed by use of the first strategy. The algorithm can choose trapezoidal shape or parallelogram shape conducting strips by use of different values for the length of inner via (parameter $d$ in Fig. 1) and the length of outer via (parameter $a$ in Fig. 1). Trapezoidal shape strips lower the resistance but increase the parallel capacitance $c_{\mathrm{p}}$. One disadvantage of the current algorithm version consist in the fact only to be able to compare I-inductors with two magnetic cores.

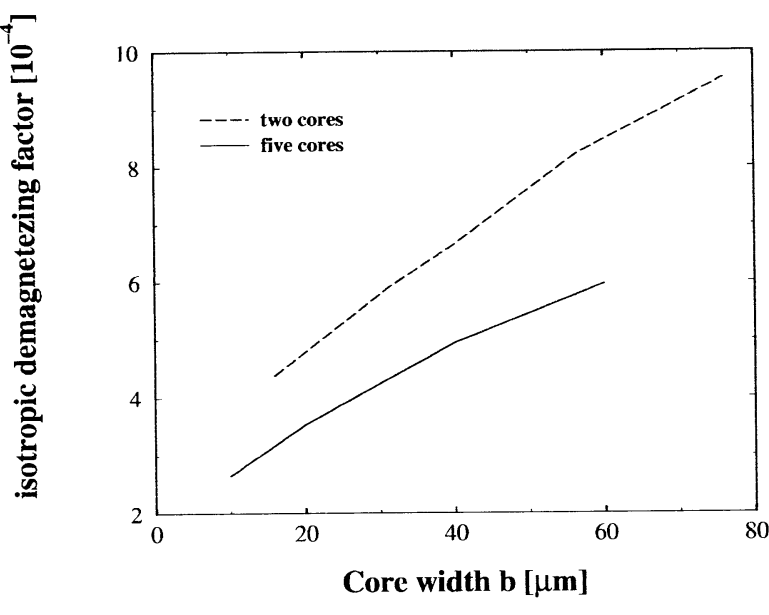

Fig. 4 Demagnetizing factor versus core width for a different number of cores.

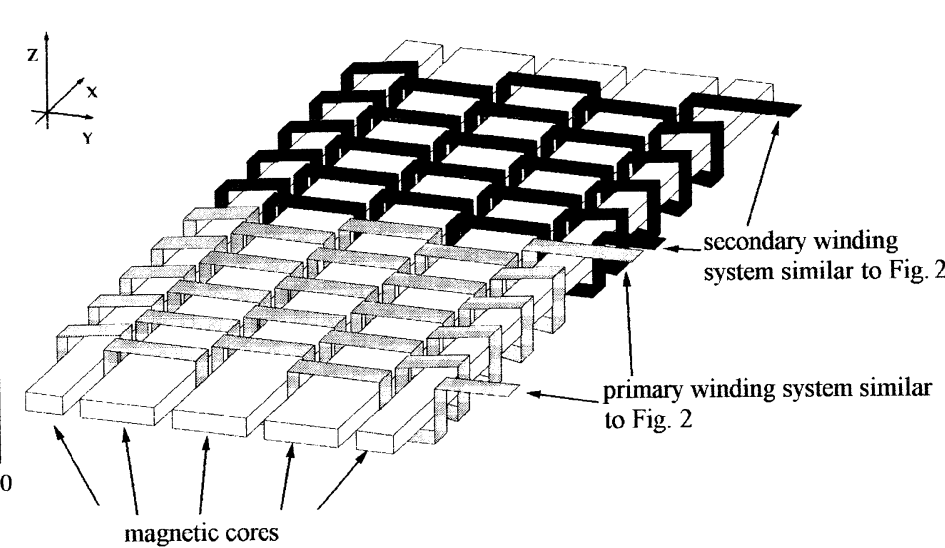

Fig. 5 Conventional design of a micro transformer basing on two adjacent five strips I-inductors. 


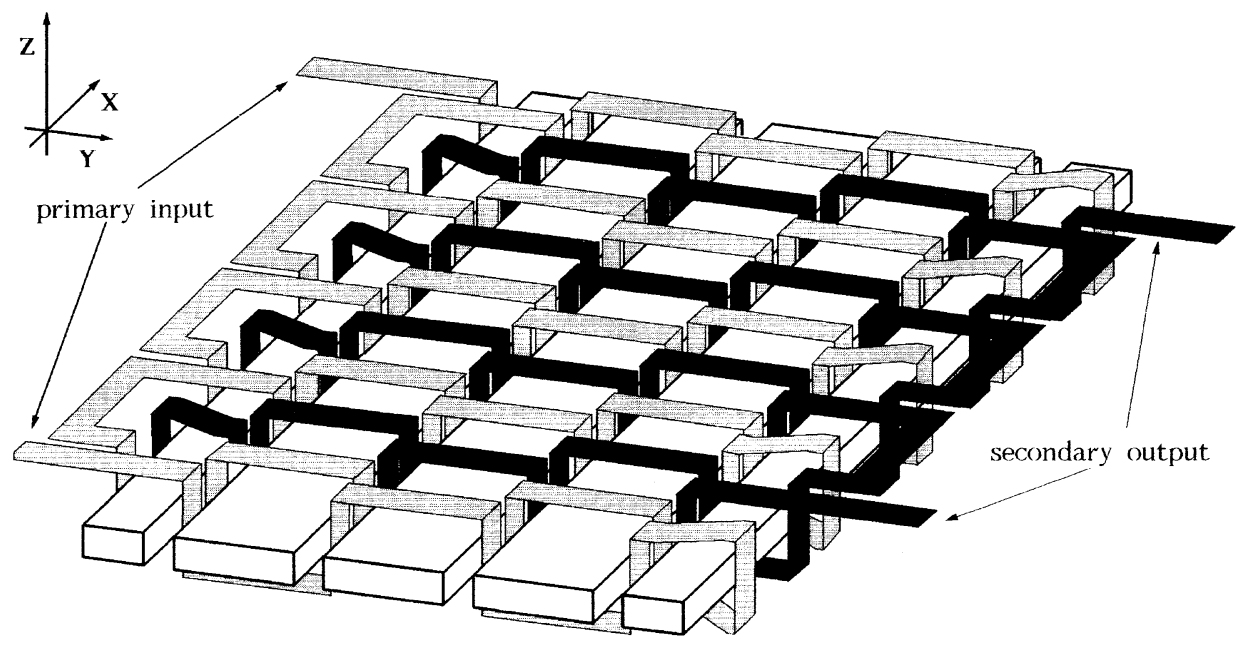

Fig. 6 Sketch of a multi chamber version of a micro transformer.

\subsection{Procedure to compare different inductor types}

One proposal of this paper is a comparison between different inductor types. In the case of the I-inductor or trench type inductor there is no answer to the question how many magnetic cores are necessary for the "best" I-inductor. The answer may be of course dependent on the application respective the target inductance. Such a comparison is performed in this paper by a two cores based I-inductor and a five strips based I-inductor. Both types are designed in the way that the magnetic flux coming out of any core is received approximately by adjacent cores. This causes in the five strips I-inductor of Fig. 2 to reduce the widths of the outside located cores. The structure of the five strips I-inductor is a little bit more complicated than the former one. Therefore other approximation of the demagnetizing factor with regard to the inductance estimation is needed. For instance a good approximation of the anisotropic demagnetization factor $\left.{ }^{2}\right)$, Fig. $4\left(d_{\mathrm{s}}=2 \mu \mathrm{m} l_{\mathrm{x}}=500 \mu \mathrm{m}\right)$, of a two cores based inductor uses a polynomial of order two, a polynomial of order four has to be used in the case of a five strips based inductor.

\section{Micro transformer}

This paper contains as a second topic a first proposal for micro transformer based on the experiences of the former simulations: Central part of a micro transformer is the parallel strips or cores of a magnetic uniaxial anisotropic material. To begin with it is necessary to design at least two magnetically coupled coils (a mutual inductance coupling) with more than $5 \mathrm{nH}$ coupling inductance basing on the five strips I-inductor in Fig. 2. It has the advantage to reach a high inductance and there is more area for the winding systems than in the case of a two strips based system. The paper will discuss two modifications of such a micro transformer: A conventional winding system with two "chambers" one for the primary coil and the other one for the identical secondary coil. The second modification is called a multi chamber system, see Table 1 . In this case there had been placed alternating one winding strip of the primary coil and one winding strip of the secondary coil, see Fig. 6. The shape of the magnetic cores is held constant. This setup is evoked by the idea to posses a primary winding strip at the fronts of the cores. This lowers the number of secondary windings (one winding strip of the secondary coil taken away) and increases the width of the conducting strips. The self inductance is calculated as written in chapter 3.1 of this paper. The coupling inductance is estimated by the sum of the flux changes in all windings of the secondary winding system caused by an exciting current change in the primary coil. This procedure uses the numerical solution of the Maxwell's equations for the self inductance from MAFIA.

\section{Results}

\subsection{Two diagrams of the maximum possible quality} factor

A genetic algorithm yields a large number of modifications for one type. Therefore these modifications have to be graded for the total width of the device keeping in mind the different number of strips. If the algorithm do not found a fitting solution within $4 \times 10^{6}$ try, then a quality factor of zero is indicated. Fig. 7 shows the maximum quality factor for a two strips based I-inductor as shown in Fig. 1 designed for three target inductance. The values $1 \mathrm{nH}$, $2 \mathrm{nH}$ and $10 \mathrm{nH}$ are typical trim inductance for wireless application. The two strips based I-inductors, Fig. 7, show two groups of solutions. On the left side are high quality factor solutions with a total width of the application less than $50 \mu \mathrm{m}$. On the other side solution groups with a larger total width but less quality factor are observed. Use of anisotropic non linear material model ${ }^{2)}$ together with material properties for a FeCoBSi alloy ${ }^{5)}$ identifies the 


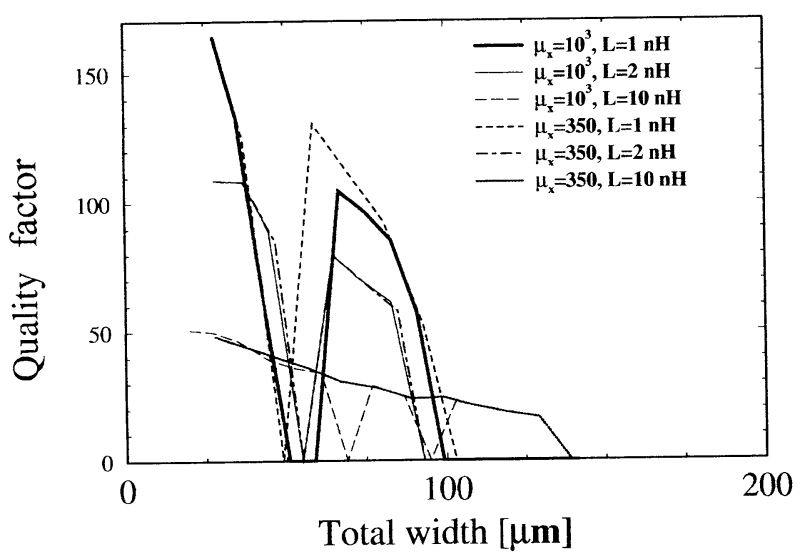

Fig. 7 Maximum possible quality factor at $0.9 \mathrm{GHz}$ versus total width of the two strips I-inductor.

additional property of the solution groups: The high quality factor inductors (core width $b=10 \mu \mathrm{m}$ core length $l_{\mathrm{x}}$ about $900 \mu \mathrm{m}$, total width $B$ of a two strips design is equal to $2(b+e)+2 \mu \mathrm{m})$ can only work with a pulsed current of about $300 \mathrm{~mA}$ caused by a saturation of the cores, while the other inductor design enable up to $800 \mathrm{~mA}$ pulsed current. A permeability larger than 100 restricts of course the maximum possible quality factor, but it is always possible to find a solution with a similar quality factor by use of other geometrical dimensions. A collapse of the quality factor is observed for permeability less than 100 for this type of inductor. The results of the five strips based I-inductor differs from those results. In Fig. 8 group behavior is not observed. This means, the algorithm find nearly for the whole range of the core width $b$ solutions with the target inductance. Except the case of $\mu_{x}=1000$, the algorithm do not find a solution for a target inductance of $1 \mathrm{nH}$. That is why a five strips I-inductor posses at least five winding. A small increase of the core width $b$ will turn the inductance to higher values than $1 \mathrm{nH}$. Next significant difference to Fig. 7 is that a five strips I-inductor stipulates a high permeability magnetic core material. Otherwise the quality factor collapses. This can be manifested by the lower demagnetizing factor of a five strips based setup, Fig. 4. It can be proofed that the part of the inductance, whose origin is the magnetic core system, increases to an optimum ${ }^{6}$, if the permeability is approximately equal to the inverse of demagnetizing factor. This optimum is caused by the influence of eddy current excitation prevention on the thickness of the magnetic cores $d_{\mathrm{s}}$. A five strips based I-inductor do not achieve a higher quality factor as a two strips I-inductor for all three target inductance. These inductors will own a higher inductance, a higher ohmic resistance and increased capacitances, but the resistance and capacitance are "growing" faster than the inductance.

This property is caused by the additional trenches in the magnetic material together with a higher number of vias.

5.2 Self inductance of the two micro transformers

The self inductance of the two micro transformers selected is shown with Fig. 9. In the case of low permeability core material the multi chamber version shows

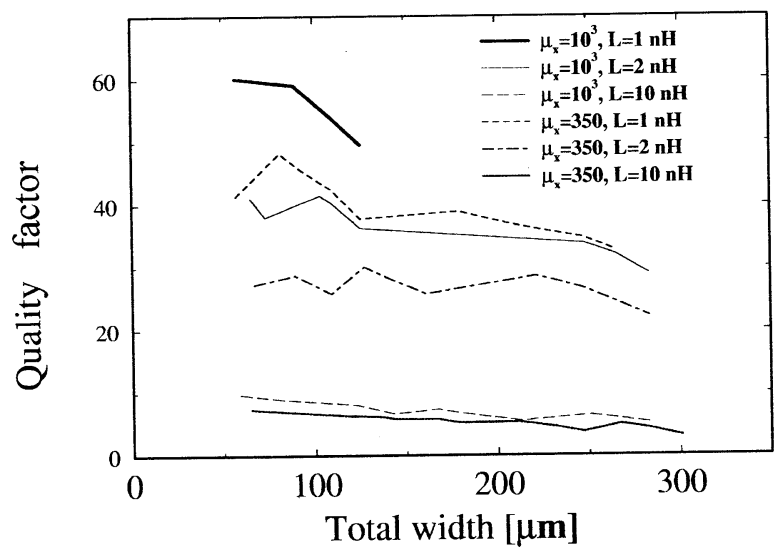

Fig. 8 Maximum possible quality factor at $0.9 \mathrm{GHz}$ versus total width of the five strips based I-inductor.

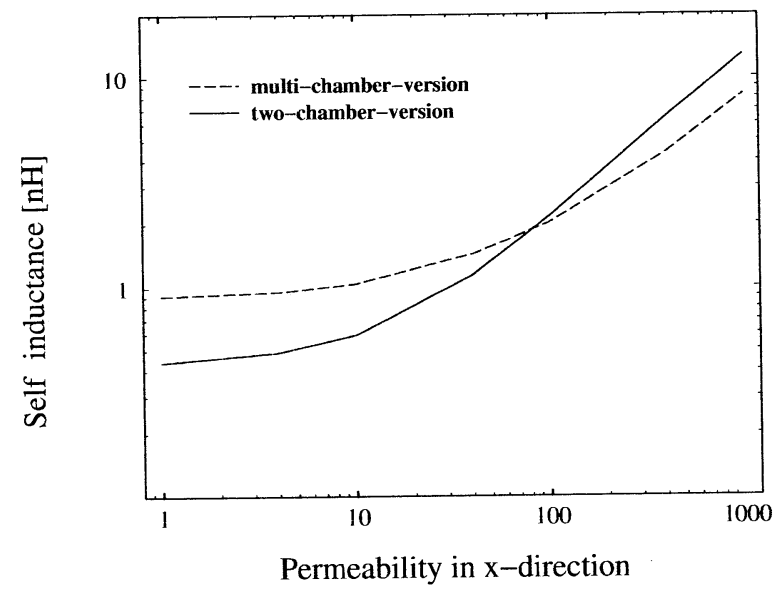

Fig. 9 Self inductance of the primary coil for different micro transformers versus permeability.

a higher inductance. This additional inductance is caused by the connections from one chamber to the other of the primary coil system. The multi chamber micro transformer from Fig. 6 shows a lower self inductance than the conventional setup from Fig. 5 for the technical interesting casc of a high permeability. This feature must not be a disadvantage. The larger area used for the primary coil system enables this feature. It causes in future transformer calculations to consider that a part of the self inductance estimated here does not couple magnetically with the secondary coil. The most important result of this section is shown with Fig. 10 by the coupling inductance. In the whole range of the simulated permeability the multi chamber version shows a higher coupling inductance per turn. Even the coupling inductance is higher, the number of turns is lowered to a $80 \%$ smaller value, see Table 1 micro transformers. This strange behavior is regarded to the anisotropy of the magnetic core material. The solutions of the Maxwell's equations under consideration of an isotropic magnetic material can described completely by the magnetic field of surface currents ${ }^{8)}$ regarding to the boundary elements method. But an anisotropic magnetic 
Table 1 Parameters of micro transformers.

\begin{tabular}{l|c|c}
\hline & Conventional setup & Multi chamber version \\
\hline Core dimensions, see fig. $1, b \times l_{\mathrm{x}} \times d_{\mathrm{s}}[\mu \mathrm{m}]$ & $20 \times 560 \times 2$ & $20 \times 560 \times 2$ \\
\hline Width of conducting strips $[\mu \mathrm{m}]$ & 23 & 25 \\
\hline Ohmic resistance: Primary coil / secondary coil $[\Omega]$ & $1.2 / 1.2$ & $1.1 / 0.9$ \\
\hline Number of turns: Primary coil / secondary coil & $25 / 25$ & $25 / 20$ \\
\hline Primary coil self inductance $\left(\mu_{\mathrm{x}}=1000\right)[\mathrm{nH}]$ & 13 & 8.2 \\
\hline Coupling inductance $\left(\mu_{\mathrm{x}}=1000\right)[\mathrm{nH}]$ & 0.7 & 5.9 \\
\hline
\end{tabular}

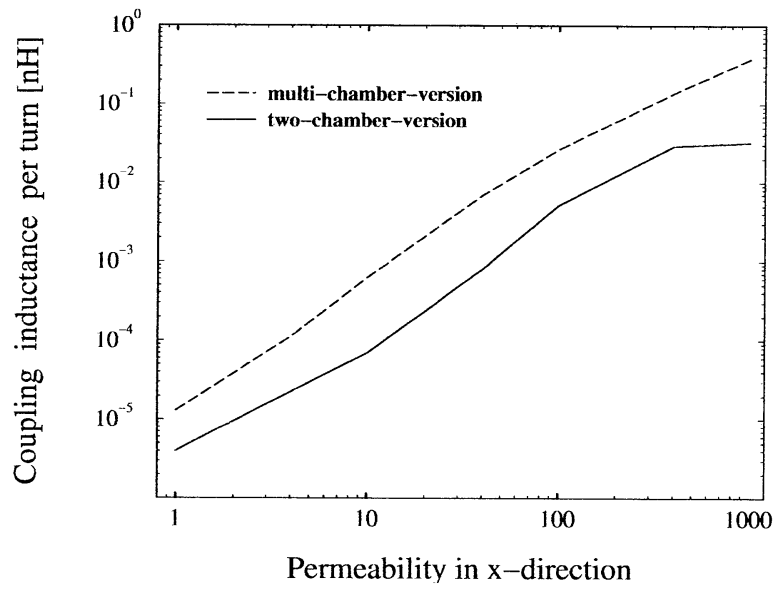

Fig. 10 Coupling inductance of different micro transformers versus permeability.

material increases additional parts in the magnetic field, which have to be characterized by so called volume currents inside the magnetic cores. This lowers the distance of the influence of the magnetic field inside the cores dependent on the permeability. A low permeability lowers the magnetic flux density in the cores as a second reason. Therefore the windings from primary coil system and the secondary coil system have to be mostly close or entwined together. The material permeability has a doubled influence on the coupling inductance. An usual toroidal transformer with an isotropic magnetic core material indicates a linear dependence for the coupling inductance on the permeability. This difference to conventional transformers forces possibly other circuits for the proposed micro transformer.

\section{Conclusion}

This paper compares a two and a five strips based I-inductor with the result that the two strips based type will theoretically achieve an higher quality factor. Future investigations should clarify the core shape dependence of the ferromagnetic resonance frequency ${ }^{6), 7)}$. Their results might cause a five strips based I-inductor type. The simulations of a micro transformer demonstrate the predominance of the multi chamber design. An application as a part of a new inductive coupled voltage controlled oscillator might be possible. This motivates to future research on this topic and numerical optimization of a multi chamber micro transformer.

\section{Acknowledgement}

This work has been performed at Forschungszentrum Karlsruhe within the framework of the collaborative resarch projekt "Material und Verfahrensentwicklung für mikrotechnische Hochleistungsbauteile (MALVE) of the Hermann von Helmholtz-Gemeinschaft Deutscher Forschungszentren (HGF). We reciprocate to all colleagues in this project for discussion and stimuli.

\section{References}

1) D. Gardner, A. M. Crawford, S. Wang: IEEE International Interconnect Technology Conference, 101 (2001).

2) A. von der Weth, J. Aktaa: J. Magn. Magn. Mat., 234, 556 (2001).

3) M. Yamaguchi: Handbook of thin film devices, 4, 185 (2000).

4) V. Korenivski: J. Magn. Magn. Mat., 215-216, 800 (2000).

5) I. Fergen, K. Seemann, A. von der Weth, A. Schüppen: $J$. Magn. Magn. Mat., 242-245, 146 (2002).

6) A. von der Weth, J. Aktaa: J. Magn. Magn. Mat., 242-245, 1206 (2002).

7) O. Geradin, J. B. Youssef, H. Le Gall : J. Appl. Phy., 88(10) 5899 (2000).

8) A. von der Weth, R. Becker: J. Nucl. Instr. and Meth. A 427 , 309 (1999).

(Received June 7, 2002; Accepted September 11, 2002) 